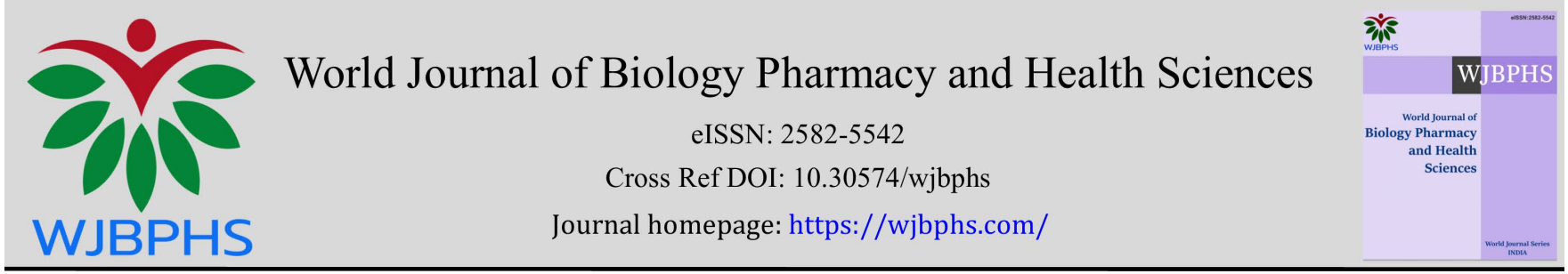

(REVIEW ARTICLE)

\title{
Covid-19 vaccination: The pros and cons
}

\author{
Odangowei Inetiminebi Ogidi $1{ }^{1}{ }^{*}$, Wonyinbarakemi Ladi Berefagha ${ }^{2}$ and Ebifanimi Okara ${ }^{3}$ \\ ${ }^{1}$ Department of Biochemistry, Federal Polytechnic Ekowe, Bayelsa State, Nigeria. \\ 2 Department of Maternal and Child Health Nursing, Niger Delta University, Wilberforce Island, Bayelsa State, Nigeria. \\ ${ }^{3}$ Research Laboratory, Federal Medical Center, Yenagoa, Bayelsa State, Nigeria.
}

World Journal of Biology Pharmacy and Health Sciences, 2021, 07(01), 015-022

Publication history: Received on 02 June 2021; revised on 05 July 2021; accepted on 08 July 2021

Article DOI: https://doi.org/10.30574/wjbphs.2021.7.1.0072

\begin{abstract}
The advent of Severe Acute Respiratory Syndrome Corona Virus 2 (SARS-CoV-2) also known as COVID-19 disease and the dynamics of its rapid spread around the globe are unprecedented. Different preventive efforts have been undertaken in response to this global health challenge, amongst them, vaccine development, distribution and dispensation is at the forefront. Vaccines stimulate the body's immune system against infectious pathogens; hence, they are one of the greatest medical accomplishments and a cornerstone of public health. There is a strong consensus globally that COVID-19 vaccine is likely the most effective approach to sustainably controlling the COVID-19 pandemic. An unprecedented research effort and global coordination has resulted in a rapid development of COVID-19 vaccines. Here, we review the various types, Pros (status of different COVID-19 vaccines, their utility in COVID-19 control and as a tool of herd immunity or protection) and Cons (various side effects, leaky vaccination and vaccine hesitancy) of COVID-19 vaccines. Despite all cons it is believed that vaccination will certainly help in building up of herd protection against COVID-19 disease, which could allow lockdowns, travel restrictions and social distancing to be relaxed globally.
\end{abstract}

Keywords: COVID-19 disease; Vaccine; Pros; Cons; Immunity; Public health

\section{Introduction}

The rapid spread of Severe Acute Respiratory Syndrome Corona Virus 2 (SARS-CoV-2) throughout the globe poses a daunting public health emergency. Different preventive efforts have been undertaken in response to this global health predicament, amongst them, vaccine development is at the forefront. Vaccines stimulate the body's immune system against infectious pathogens; hence, they are one of the most effective ways of preventing diseases [1].

The immune response to the SARS-CoV-2 involves innate immune activation and antigen-specific responses of bone marrow or bursa-derived (B) and thymus (T) cells. Protection from viral infection is mainly achieved by virusneutralizing antibodies, a principle that applies to the vast majority of viral infections to which humans acquire robust immune protection due to infection or vaccination. Broad immunization has been very helpful as soon as "only" approximately 60-70\% have become immune, because relatively simple measures against viral spread will then be sufficient to contain the virus [2].

After global approval of COVID-19 vaccines, COVID-19 has become the $2^{\text {th }}$ vaccine preventable human disease [1]. Not all infectious diseases can be presently fought with a vaccine. The HIV, respiratory syncytial virus and the cancer causing Epstein-Barr virus kill millions of people every year due to the lack of vaccines. Moreover, not everyone is fortunate enough to be vaccinated due to several economic, social, ethical, geographical and health reasons leading to vaccine preventable deaths. The health reasons for avoiding vaccines include: (1) historical evidences of severe or life-

${ }^{*}$ Corresponding author: Odangowei Inetiminebi Ogidi

Department of Biochemistry, Federal Polytechnic Ekowe, Bayelsa State, Nigeria.

Copyright (C) 2021 Author(s) retain the copyright of this article. This article is published under the terms of the Creative Commons Attribution Liscense 4.0. 
threatening reaction to the vaccine (allergies), (2) infants younger than six months old (for many vaccines), (3) ongoing moderate or severe illness and (4) people with chronic wasting diseases or those with Guillain-Barre syndrome (characterized by difficulties with eye muscles and vision, swallowing, speaking, chewing, lack of sensations in the hands and feet but severe pain especially at night, problems of coordination and steadiness beside arrhythmic heart beat and disturbed blood pressure) [3].

The most common flu shots are not suggested be administered to children under two years of age, or young children with a history of asthma or wheezing, children or adolescents on long-term aspirin treatment and pregnant women. Also, people with chronic diseases, (such as heart disease, liver disease, or asthma), people with certain muscle or nerve diseases (that can cause breathing problems) and people who have (or are living with family members) compromised immune systems are advised against the flu vaccine. The vaccine shots usually fail to protect elderly from common flu. However, there is paucity of information about COVID-19 vaccine suitability, which may be learnt after several complications yet to be reported after these mass scale vaccinations around the world [3].

As of 10 April 2021, the top five countries with vaccination programs are the United States (6.129 million), China (4.052 million), the European Union (2.66 million), the United Kingdom (1.82 million) and India (1.084 million) [4]. Although the implementation of vaccination is one of the important factors to achieve global herd immunity, there is no consensus concerning the superiority of one vaccine over the others in terms of protective efficacy and safety profile [5, 6].

To date, there are 86 vaccines under development in clinical phase trials. They are developed with different methods such as protein subunits, inactivated virus, DNA-based vaccine, RNA-based vaccine, viral vectors, and live-attenuated viruses [7], however, many of them are currently in preclinical or phase one trial.

\section{Types of vaccines}

Preventive and therapeutic are two groups of vaccines, a vaccine can belong to both of the groups depending upon the way it is designed. Vaccines are primarily used for disease prevention and outbreaks aiming to control and eradicate various diseases. Therapeutic vaccines are used mostly for diseases with a long course or slow progression but usually of high fatality like rabies, cancers (antigen vaccine), dendritic cell vaccine (still under research), autologous and allogeneic vaccine (for certain tumors). Some therapeutic vaccines for HIV and Alzheimer's disease are in experimental stage with promising outcome [8].

\subsection{Types of COVID-19 vaccines}

The potential vaccines for COVID-19 either approved for use or in trial stage (Figure 1A and B) include:

\subsubsection{Inactivated or weakened virus vaccines}

The inactivated or weakened virus is incapable of causing disease, but induces a protective immune response (CoVaxin from Bharat Biotech with claimed 70.4\% efficacy, India, Sinovac Biotech vaccine from China with more than 50 percent efficacy).

\subsubsection{Protein-based vaccines}

Subcomponent vaccines contain harmless fragments of the virus like spike proteins or protein shells that mimic the COVID-19 virus, also known as ghost viruses or virus like particles (VLPs) are often safe and generate an immune response (EpivacCorona vaccine from Russia).

\subsubsection{Viral vector vaccines}

Use a harmless career virus either natural or genetically engineered designed to carry genes to produces Corona-virus proteins in host to generate an immune response (Gam-Covid-Vac or Sputnik V from Russia with 92\% efficacy, AstraZeneca Oxford Covisheild with claimed 90\% efficacy, both used Adenovirus as vector for Covid-19 spike proteins)

\subsubsection{RNA and DNA vaccines}

A cutting-edge approach that uses genetically engineered mRNA or DNA to generate a protein, often requiring a potent delivery system and adjuvant to induce the immune system to mount a protective response. Moderna (mRNA 1273) and Pfizer (BNT162b2) vaccines with 94-95\% efficacy. 


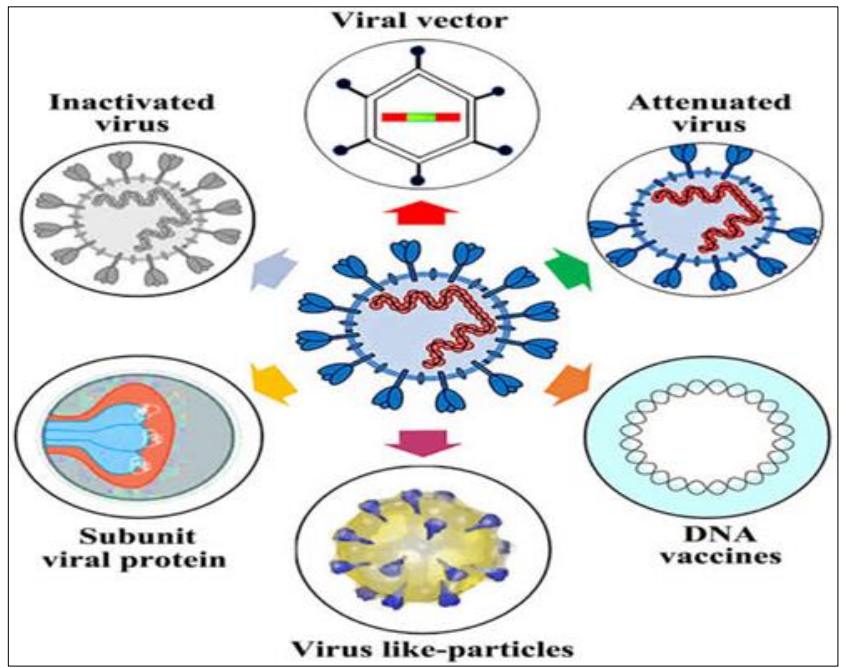

Figure 1 (a) Types of COVID-19 vaccines

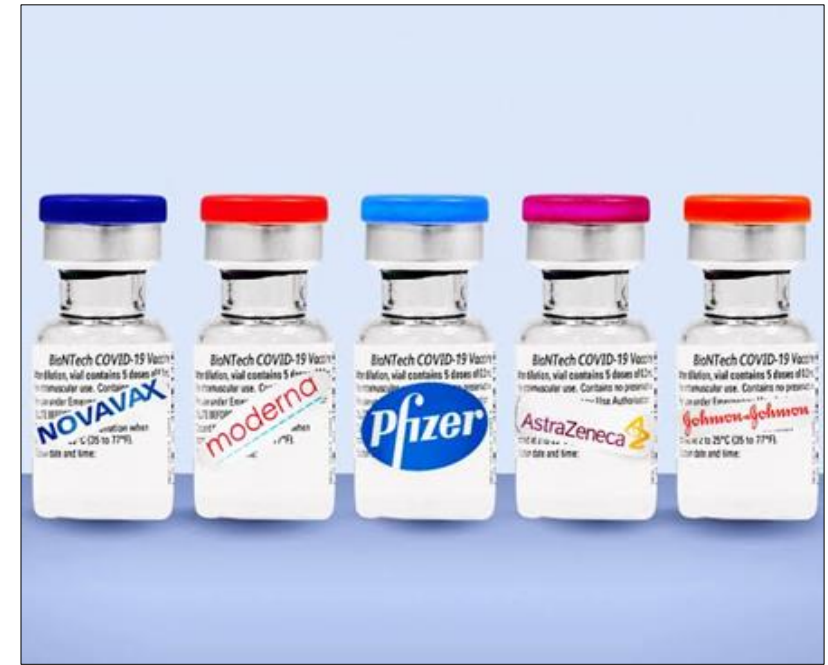

(b) WHO approved COVID-19 vaccines [9]

\subsection{Mechanism of COVID-19 vaccines}

Protection induced by currently available vaccines against viruses is primarily based on virus-neutralizing antibodies. Such antibodies usually block the interaction of the virus with its cellular receptor or prevent conformational changes required for fusion of the virus with the cell membrane. Recent investigations have shown that the new SARS-CoV-2 virus uses a similar strategy of SARS-CoV-1 in cell entry [10].

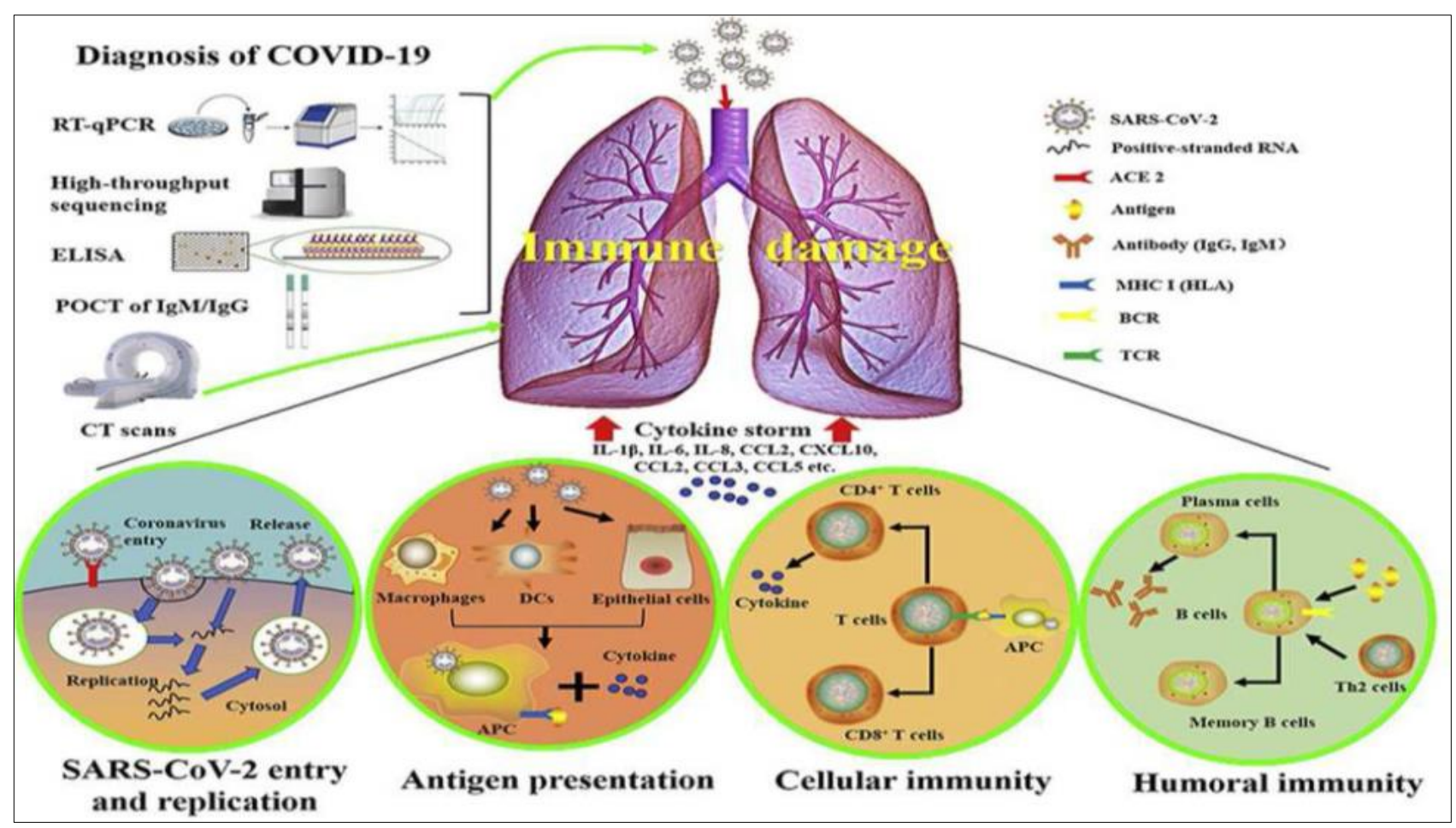

Figure 2 (a) Mechanism and diagnosis of 2019 novel coronavirus (2019-n-CoV) infection [16]

Attachment to host cells takes place via binding of the viral S protein (Figure 2A and B) to the angiotensin-converting enzyme 2 (ACE2), (the viral receptor on host cells). Subsequently, the S protein is primed by host cell proteases using furin and the serine proteases (TMPRSS2 and TMPRSS4), enabling the fusion of viral and cellular membranes and the consequent entry of viral RNA into the host cell [11].

S protein interaction with ACE2 is well described for both SARS-CoV-1 and -2 and relies on a particular domain within the $\mathrm{S}$ protein, the so-called receptor binding domain (RBD). Indeed, most antibodies capable of neutralizing 
coronaviruses are directed against RBD [12]. Hence, the primary immune mechanism of avoiding infection is through blocking viral attachment to ACE2. Therefore, generating a vaccine inducing antibodies against RBD is the strategy used by the majority of COVID-19 vaccine candidates [13]. It has recently been shown that RBD is glycosylated and methylated. Generally, such posttranslational modifications are difficult to reproduce in vaccines, meaning that vaccines may display (slightly) different epitopes than the virus. Consequently, the antibodies induced by the vaccines may potentially be cross-reactive and non-protective. Interestingly, however, the receptor interaction site (RIS) directly binding to ACE2 is not glycosylated, indicating that this RIS may potentially be an ideal vaccine candidate [14, 15] (Figures $2 \mathrm{~A}$ and $\mathrm{B}$ ).

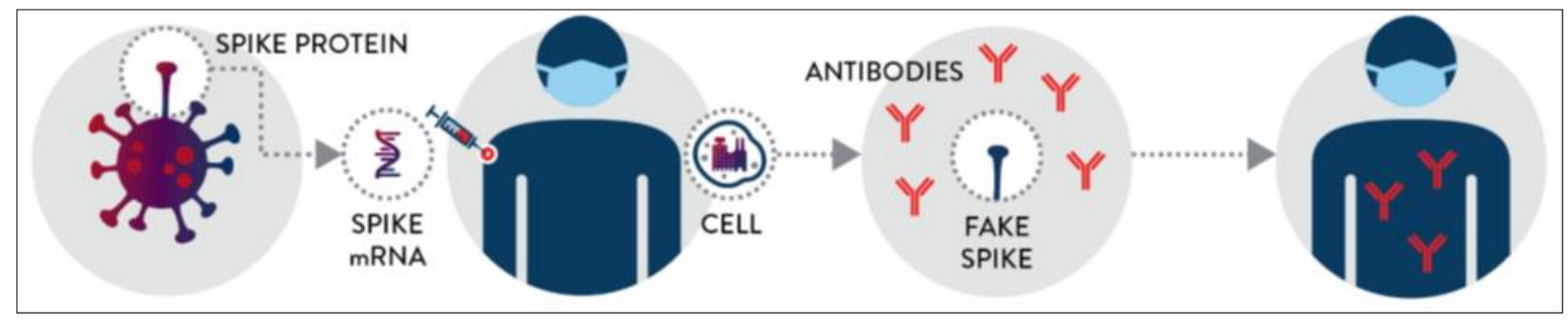

Figure 2 (b) A simplified analog of the mechanism of COVID-19 vaccines against the Virus [9]

\section{The pros}

COVID-19 vaccination will be an important tool to help stop the pandemic. Wearing masks and physical distancing help reduce the chances of being exposed to the virus or spreading it to others, but these measures are not enough. The combination of getting vaccinated and following Center for Disease Control and Prevention's (CDC) recommendation to protect ourselves and others will offer the best protection from the pandemic. The target of vaccination is to build up herd immunity, where enough of the population is protected from the virus and as a result transmission slows down significantly to kill the disease. However, vaccinologists are not sure about the magic number (fraction of immune population) to obtain effective herd immunity for COVID-19, they estimated around $70 \%$ of the population, which could take months (in nations with developed health system) to years to achieve the target through vaccination. This is certain that it is a very conservative estimate and is certain to change depending on the contagiousness of the present COVID19 and future mutant viruses destined to emerge at the face of vaccination [17].
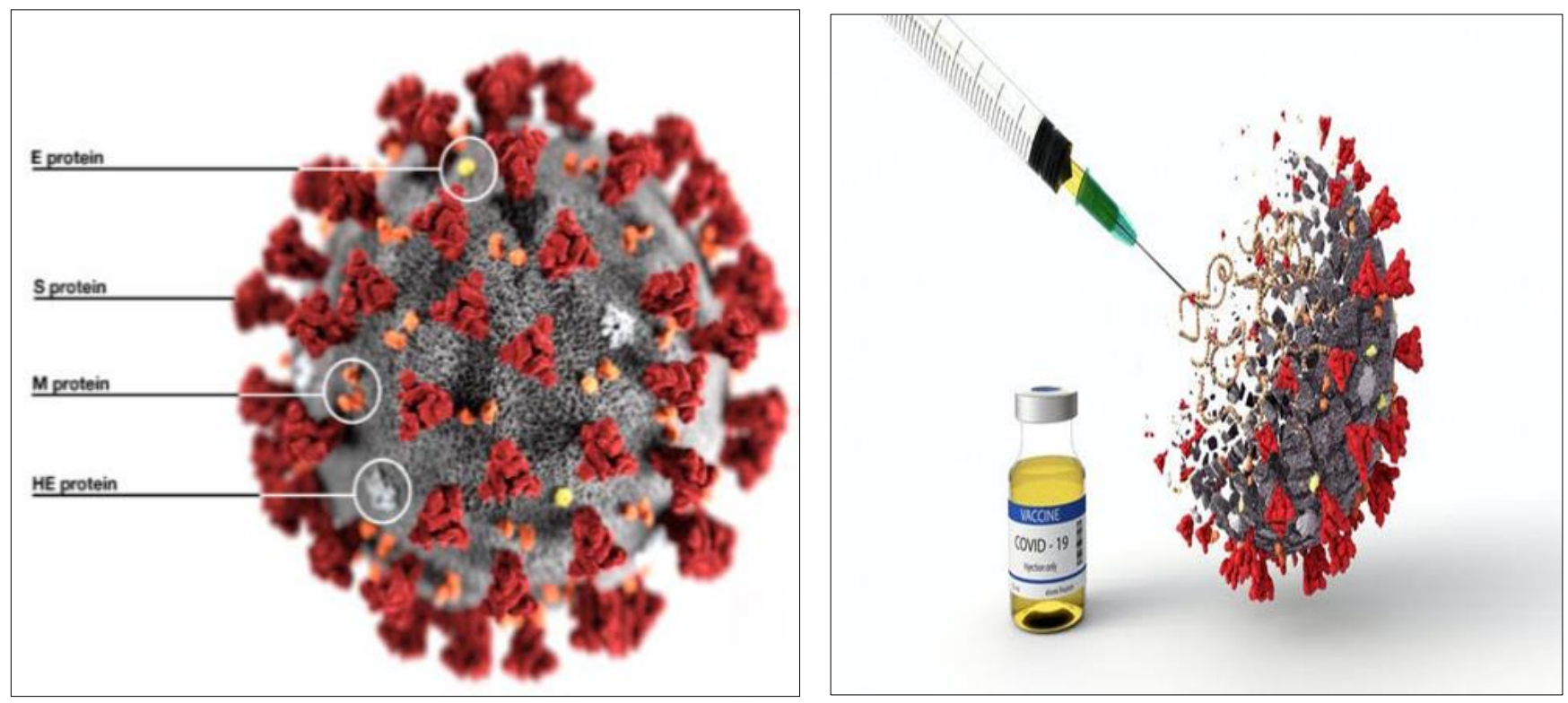

Figure 3 (a) Schematic structure of CoVs [18], (b) Structure showing the effect of COVID-19 vaccine on CoVs [9]

\subsection{COVID-19 vaccines protection}

It is quite early to know and claim if COVID-19 vaccines will either provide long-term protection or not but at present it is claimed to protect for $6-8$ months after a booster vaccination. This is an assumption and based on the available data 
suggesting that most persons who recovered from COVID-19 developed protective antibody titers (based on which serum therapy has been practiced in many nations using convalescent serum) and cellular immune response which provides protection from reinfection for 6-8 months [19].

\subsection{Efficacy of COVID-19 vaccines}

The Center for Disease Control and Prevention (CDC) pointed out, "We do not know what happens after a single dose," William Moss (director of the International Vaccine Access Center) says. "Certainly we cannot expect (that one dose will confer) the high degree of protection" that both doses demonstrated in phase 3 clinical trials. (Pfizer's vaccine, for example, was found to be effective at 52 and 95 percent after the first and second doses respectively). An interesting finding from the Pfizer- BioNTech and Moderna clinical trials is that the vaccines were effective in older adult participants with minimal side effects when compared to the younger participants [17]. However, the efficacy of COVID19 vaccine is far better than the effectiveness of the annual flu shot (40\%).

\subsection{Natural immunity after COVID-19 infection versus COVID-19 vaccine immunity}

People after COVID-19 infection (those had minor ailment after infection or remained even asymptomatic) develop fairly good and long-lasting immune responses. COVID-19 antibodies lasted at a good level even after 6-8 months of infection. Thus it is envisaged that long-lasting antibody levels and immune memory cells could potentially lessen the severity of a re-infection [19]. The immunity after the first infection leads to floating of an idea for an "immunity passport" or "risk-free certificate" that would enable individuals to travel or to return to work assuming that they are protected against re-infection. However, the availability of limited data evidences questions the possibility of any such immunity passport. Till today, no country permits a person to travel internationally with good antibody titer report but requires a reverse transcription polymerase chain reaction (RT-PCR) based antigen detection test report.

The new vaccines were $90 \%$ and $94.5 \%$ effective, Paul said, but "naturally acquired" COVID-19 immunity was even better, at 99.9982\% efficacy [20]. However, a natural infection may not be the choice for getting immune because many of those (one in three) people who recover from COVID-19 have chronic complaints, including exhaustion and a racing heart, for months afterwards including people under 35 years of age with no previous health conditions. Several of the survivors of COVID-19 have reported symptoms similar to those of lupus and rheumatoid arthritis. COVID vaccines, in contrast, carry only a few and a little known risk. So far COVID-19 vaccines have been tested in tens of thousands of people and used in a million of people with no serious side effects [21]. The continued spread of this virus highlights the importance of global acceptance of the developed vaccines and therapeutics, for vaccination provides some degree of immune protection which could allow lockdowns and social distancing to be relaxed. The developed COVID-19 vaccines do not contain the live virus that causes COVID-19; therefore, it will not cause positive COVID-19 viral tests.

\section{The cons}

It is important to investigate whether vaccines confer full or partial protection, if this varies with age, whether vaccinated individuals are protected from recurrent infections and whether the vaccination will require boosting (which would necessitate the manufacturing of billions of additional doses). Critical potency testing, standardization and quality control of vaccines are required to determine the level of immune response. With the recent development of new technologies in vaccine manufacturing processes, it will be easy to prove that a vaccine is safe and effective.

\subsection{Reported side effects of COVID-19 vaccines during their trials}

Participants of several COVID-19 vaccine trials have experienced mild, lingering and in some cases, 'totally strange' sideeffects. Moderna trials detailed, suffering from bone breaking chills, and a fever that went up to $102^{\circ} \mathrm{F}$ within hours when vaccines were inoculated. Onset of production of antibodies may also cause a low grade or high fever, accompanied by redness and swelling at injection site [22]. Commonly reported side effects of the Pfizer- BioNTech COVID-19 vaccine include fatigue (63\%), headache (55\%), and muscle pain (38\%). These side effects were observed to last in a day or two. However, the most commonly reported (by 84\%) side effect from the vaccine is an injection site reaction, where the arm was observed to be a little tender and slightly difficulty in moving. Some participants in trials reported chills, joint pain, or fever following the vaccination. Reactions were more often reported following the second (booster) dose of the vaccine.

Serious side effects such as Bell's palsy which is characterized by rapid onset of mild weakness to total paralysis on one side of the face (facial hemiplegia, the $7^{\text {th }}$ nerve inflammation) within hours to days of the vaccination. This also leads to facial droop and difficulty in making facial expressions, such as closing of eyes or smiling, drooling and pain around the jaw or in/behind the ear have been reported in $0.5 \%$ of the vaccine trial participants [23]. 
Anaphylaxis, a potentially life-threatening allergic reaction, has been linked in the past to multiple types of vaccines. Severe allergic reaction after receiving the Moderna and Pfizer vaccines (1.31 per million doses) alerted authorities to closely see whether anaphylaxis is linked to all COVID-19 vaccines, or merely those like the Pfizer and Moderna vaccines made of messenger RNA. Eight people in the Pfizer and Moderna trials had Bell's palsy after receiving the vaccine. Having Guillain Barré syndrome is another possibility after getting vaccine shots [24]. The allergic reactions after vaccine shots as per scientific investigation may be due to the compound polyethylene glycol (PEG), which is part of the fatty envelope that surrounds the mRNA, the main ingredient in the vaccine [25].

\subsection{Leaky vaccination}

Another worry is "Leaky" vaccination. When a vaccinated host keeps on shedding disease germs due to persistence of disease causing agent without any apparent clinical disease, it is called leaky vaccination. Studies on COVID-19 vaccines at present are silent over this point. Many veterinary and human vaccines are classified as leaky because they only stop the clinical occurrence of disease but do not protect vaccinated person from getting infected or being carrier or disease disseminator. The leakiness is of concern because it increases vaccination coverage required to prevent disease spread and can also promote the evolution of the pathogen with increased virulence [3].

\subsection{Vaccine hesitancy}

Vaccine hesitancy refers to delay in acceptance or refusal of vaccines despite availability of vaccination services. Vaccine hesitancy is complex and context specific, varying across time, place and vaccines. It is influenced by factors such as complacency, convenience and confidence. In the case of COVID-19, the hesitancy may be exacerbated by the accelerated testing and approval process which applies not only to Sputnik V (the controversial "Russian vaccine") [26]. Vaccine hesitancy has existed ever since vaccines and vaccination programs began, recent acknowledgement that it can influence the impact of vaccination programs has highlighted the need to understand and address it more systematically. Vaccine hesitancy is a complex behavioral phenomenon. Its sentiments pose a growing problem that has become embroiled in anti-mask and anti-lockdown ideologies.

\section{Conclusion}

Vaccines are one of the greatest medical accomplishments and a cornerstone of public health. Despite all cons it is believed that vaccination will certainly help in building up of herd immunity. There are robust testing procedures in place in most jurisdictions to ensure that licensed COVID-19 vaccines are both effective and safe. It is only to acknowledge that everything, including vaccination, has risks. Perhaps the biggest challenge in the development of a vaccine for COVID-19 is to be honest about the extent of those risks and convey the limitations of confidence in safety and efficacy relative to the evidence accrued. There is an ethical balance to be struck which are, introducing a vaccine early and saving more lives from COVID-19, but risking side effects or ineffectiveness versus engaging in longer and more rigorous testing, and having more confidence in safety and efficacy, but more people dying of COVID-19 while such testing occurs. There is no magic answer, given the economic, social and health catastrophe of various anti-COVID-19 measures such as lockdown, there will be considerable pressure for the vaccination. Politics and private interests should not hamper vaccine development and deployment efforts. This gargantuan undertaking must be achieved through beneficent partnerships between industry, governments and international organizations for the universal benefit of human health.

\section{Compliance with ethical standards}

\section{Acknowledgments}

Authors express special thanks and appreciation to Prof. C. Nweke and Prof. J. N. Okereke for their inspiration on the subject and to Dr. E. A. Anyalogbu and Mr. Frank-Oputu Ayibaene for their overall support and motivation throughout the writing of this article.

\section{Disclosure of conflict of interest}

Author declares no conflict of interest.

\section{References}

[1] Singh B, Gandharava S, Gandharva R. Covid-19 vaccines and Community Immunity. Infect Dis Res. 2021 ; 2: 1-7. 
[2] Thevarajan I, Nguyen THO, Koutsakos M, Druce J, Caly L, Van-De-Sandt CE, et al. Breadth of concomitant immune responses prior to patient recovery: A case report of non-severe COVID-19. Nat Med. 2020; 26: 453-5.

[3] World Health Organization [Internet]. Global Vaccine action plan. 2020.

[4] Ritchie H, Beltekian D, Mathieu E, Hasell J, Macdonald B, Giattino C, et al. Statistics and Research: Coronavirus (COVID-19) Vaccinations. 2021.

[5] Liu X, Liu C, Liu G, Luo W, Xia N. COVID-19: Progress in diagnostics, therapy and vaccination. Theranostics. 2020; 10: 7821-35.

[6] Karpinski TM, Ozarowski M, Seremak-Mrozikiewicz A, Wolski H, Wlodkowic D. The 2020 race to-wards SARSCoV-2 specific vaccines. Theranostics. 2021; 11: 1690-702.

[7] World Health Organization [Internet]. Solidarity Vaccines Trial Expert Group. Draft Landscape and Tracker of COVID-19 Candidate Vaccines. 2021.

[8] Webmd.com. [Homepage in internet]. Frequently Asked Questions on COVID-19 Vaccine Safety, Side Effects and Efficacy. 2021.

[9] Byram WB, Shayan S, [Internet]. Fast covid-19 vaccines timelines are unrealistic and put the integrity of scientists at risk. 2021.

[10] Hoffmann M, Kleine-Weber H, Schroeder S, Krüger N, Herrler T, Erichsen S, et al. SARS-CoV-2 Cell Entry Depends on ACE2 and TMPRSS2 and Is Blocked by a Clinically Proven Protease Inhibitor. Cell. 2020; 181: 271-80.

[11] Zang R, Castro MFG, McCune BT, Zeng Q, Rothlauf PW, Sonnek NM, et al. TMPRSS2 and TMPRSS4 promote SARSCoV-2 infection of human small intestinal enterocytes. Sci Immunol. 2020; 5: 3582.

[12] Barnes CO, West AP, Huey-Tubman KE, Hoffmann MA, Sharaf NG, Hoffman PR, et al. Structures of Human Antibodies Bound to SARS-CoV-2 Spike Reveal Common Epitopes and Recurrent Features of Antibodies. Cell. 2020; 182: 1-5.

[13] Zhang J, Zeng H, Gu J, Li H, Zheng L, Zou QM. Progress and Prospects on Vaccine Development against SARS-CoV2. Vaccines. 2020; 8:153.

[14] Sun Z, Ren K, Zhang X, Chen J, Jiang Z, Jiang J, et al. Mass spectrometry analysis of newly emerging coronavirus HCoV-19 spike S protein and human ACE2 reveals camouflaging glycans and unique post-translational modifications. Biorxiv. 2020.

[15] Grant OC, Montgomery DW, Ito K, Woods RJ. Analysis of the SARS-CoV-2 spike protein glycan shield: Implications for immune recognition. Biorxiv. 2020; 9: 2754.

[16] Chitra BH, Rubiya SQ, Amol AJ. A review on covid-19- A Global Battle between Life and death. Int J Pharm Res. $2021 ; 12: 19-24$.

[17] Aarp.org. [Homepage in internet]. What Are the Side Effects of COVID-19 Vaccines?

[18] Abdullah A, Vishal SC, Satyendra PS, Satya PS, Neda A. Rise and fall in Sars-CoV-2 Global Pandemic strain rate-An Overview. Int j App Pharma. 2021; 13: 47-67.

[19] Gavi.org. [Homepage in internet]. The Vaccine Alliance.

[20] Indianexpress.com. [Homepage in internet]. Explained: Does 'natural immunity' from Covid-19 offer better protection that a vaccine?

[21] Nytimes.com. [Homepage in internet]. What if you've already had Covid-19 - do you still need a vaccine? Experts tackle questions about vaccine immunity.

[22] Timesofindia.indiatimes.com. [Homepage in internet]. Coronavirus Vaccine: 5 Side-Effects Doctors Want To 'Warn' The Public. 2020.

[23] Healthline.com. [Homepage in internet]. What We Know About the Side Effects of Pfizer's COVID-19 Vaccine. 2020.

[24] Statnews.com. [Homepage in internet]. The curse of the incidental illness: Seen as side effects to Covid vaccinations, ailments may have little to do with them.

[25] Webmd.com. [Homepage in internet]. Treating Disease with Vaccines. 2020.

[26] Savulescu J. Good reasons to vaccinate: Mandatory or Payment for risk? J Med Ethics. 2020; 2: 1-8. 


\section{Authors short Biography}

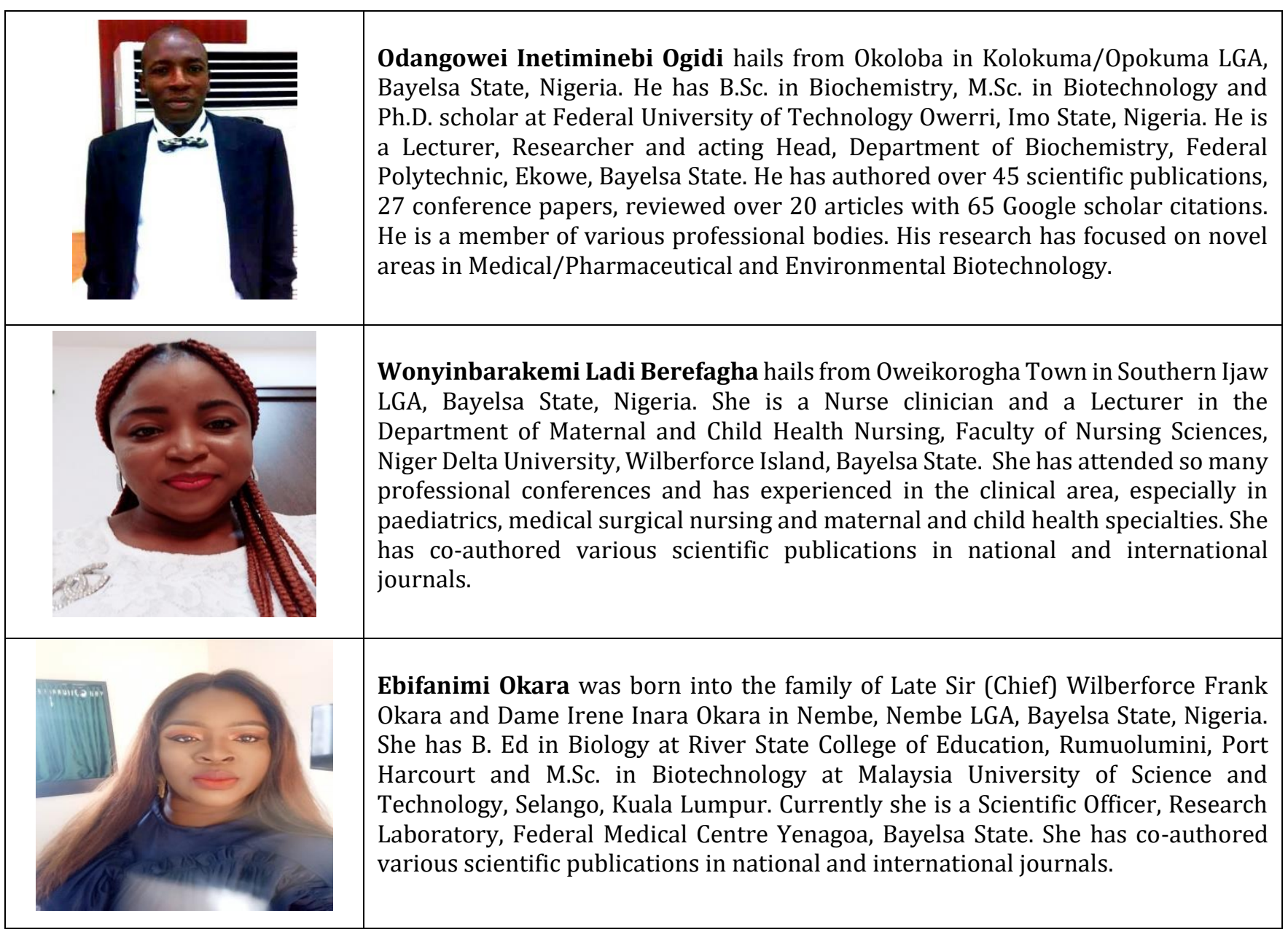

